\title{
In vivo correction of ZAP-70 immunodeficiency by intrathymic gene transfer
}

\author{
Oumeya Adjali, ${ }^{1}$ Gilles Marodon, ${ }^{2}$ Marcos Steinberg, ${ }^{1}$ Cédric Mongellaz, ${ }^{1}$ \\ Véronique Thomas-Vaslin, ${ }^{2}$ Chantal Jacquet, ${ }^{1}$ Naomi Taylor, ${ }^{1}$ and David Klatzmann ${ }^{2}$ \\ 1 Institut de Génétique Moléculaire de Montpellier, CNRS UMR 5535/Institut Fédératif de Recherch 122, Montpellier, France. \\ 2Université Pierre et Marie Curie/CNRS UMR 7087, Hôpital Pitié-Salpêtrière, Paris, France.
}

\begin{abstract}
SCID patients have been successfully treated by administration of ex vivo gene-corrected stem cells. However, despite its proven efficacy, such treatment carries specific risks and difficulties. We hypothesized that some of these drawbacks may be overcome by in situ gene correction of $T$ lymphoid progenitors in the thymus. Indeed, in vivo intrathymic transfer of a gene that provides a selective advantage for transduced prothymocytes should result in the generation of functional $T$ lymphocyte progeny, allowing long-term immune reconstitution. We assessed the feasibility of this approach in a murine model of ZAP-70-deficient SCID. A T cell-specific ZAP-70-expressing lentiviral vector was injected into thymi of adult $Z A P-70^{-/-}$mice without prior conditioning. This resulted in the long-term differentiation of mature TCR- $\alpha \beta^{+}$thymocytes, indicating that the vector had integrated into progenitor cells. Moreover, peripheral ZAP-70-expressing T cells demonstrated a partially diversified receptor repertoire and were responsive to alloantigens in vitro and in vivo. Improved treatment efficacy was achieved in infant $Z A P-70^{-/-}$mice, in which the thymus is proportionately larger and a higher percentage of prothymocytes are in cycle. Thus, intrathymic injection of a lentiviral vector could represent a simplified and potentially safer alternative to ex vivo gene-modified hematopoietic stem cell transplantation for gene therapy of $\mathrm{T}$ cell immunodeficiencies.
\end{abstract}

\section{Introduction}

SCID is a heterogeneous group of genetic disorders that is almost universally fatal in infancy, due to the advent of opportunistic infections. SCID can be treated by allogeneic stem cell transplantation, but the majority of patients do not have histocompatible donors. In the absence of histocompatible donors, SCID patients sometimes receive an HSC transplant from HLA-haploidentical donors. Although recent modifications of this protocol have increased survival to levels approaching $75 \%$, there are significant short-term and long-term complications, and the emergence of significant numbers of circulating naive $T$ cells often requires more than 150 days (1-3). Alternatively, SCID represents a unique favorable setting for gene therapy strategies as the gene-corrected lymphocytes should have a selective advantage. Indeed, such an approach has already been shown to be beneficial in several adenosine deaminase-deficient (ADA-deficient) and $\gamma c$-deficient SCID patients (4-7). The extensive ability of $\mathrm{T}$ progenitors to undergo massive expansion translates into the possibility that only a few corrected progenitor cells can reconstitute the $\mathrm{T}$ cell compartment, as highlighted by patients in whom a spontaneous reversion mutation resulted in a "natural" gene therapy. Specifically, several individuals with inherited mutations that are known to result in

Nonstandard abbreviations used: ADA, adenosine deaminase; BV, $\beta$ chain hypervariable region; CDR3, complementarity determining region 3; DN, double negative; DP, double positive; eGFP, enhanced GFP; FSC, forward angle scatter; IRES, internal ribosome entry site; IT, intrathymic(ally); PT-ZAP, T cell-specific lentiviral vector encoding human ZAP-70; SP, single positive; SSC, side angle scatter; TCRBV, TCR $\beta$ chain hypervariable region; $\mathrm{TU}$, transduction unit.

Conflict of interest: The authors have declared that no conflict of interest exists.

Citation for this article: J. Clin. Invest. 115:2287-2295 (2005).

doi:10.1172/JCI23966.
SCID (X-SCID and ADA-deficiency) have been found to be relatively healthy, with relatively normal $T$ cell numbers (8-11). In each of these patients, this was likely due to a reversion mutation, and because this event is statistically improbable, it likely occurred in a single hematopoietic progenitor/stem cell (8-10). These natural gene therapy experiments strongly suggest that gene correction of a single progenitor cell that is capable of proliferation, differentiation, and migration can potentially eliminate the critical symptoms associated with SCID.

Based on the findings that correction of a single or few progenitor cells can restore immune reconstitution, clinical gene therapy trials for SCID disorders have thus far relied on ex vivo gene transfer into HSC/progenitor cells $\left(\mathrm{CD} 34^{+}\right)$using nonspecific retroviral vectors. Despite its proven efficacy $(4,6,7,12,13)$, such an approach carries specific risks and difficulties (14). First, as gene transfer with murine leukemia virus-based retroviral vectors cannot occur in the absence of mitosis, this approach requires an ex vivo culture of $\mathrm{CD} 34^{+}$cells in the presence of a cocktail of cytokines. This is a cumbersome manipulation that can affect the long-term function of these cells. Second, the ectopic expression of the therapeutic gene in all stem cell progeny could generate serious side effects, notably when the transgene participates in signal transduction pathways. Finally, oncoretroviral insertion per se, by modifying the expression of cellular genes, can contribute to leukemogenesis $(14,15)$.

We hypothesized that some of these drawbacks could be overcome by in situ gene correction of $\mathrm{T}$ lymphoid progenitors in the thymus using T cell-specific lentiviral vectors. This hypothesis was supported by the following observations: (a) lentiviral vectors have resulted in efficient in vivo gene transfer in hepatocytes, antigen-presenting cells, and muscle cells as well as cells in the 


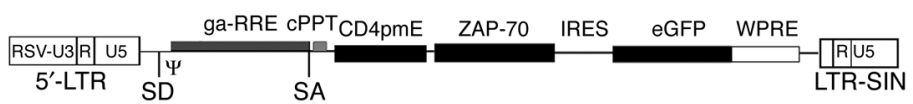

Figure 1

Schematic representation of the PCD4 lentiviral vector encoding WT ZAP-70. The relative positions of the elements contained within the pCD4 lentiviral vector are indicated. Both $3^{\prime}$ and $5^{\prime}$ long terminal repeats (LTRs) are derived from Rous Sarcoma Virus (RSV). SD, splice donor; SA, splice acceptor; $\Psi$, packaging signal; ga-RRE, truncated gag sequence with the rev responsive element; cPPT, central polypurine tract of HIV; CD4pmE, human CD4 minimal promoter/murine enhancer cassette (590 bp); ZAP-70, ZAP-70 cDNA; eGFP, eGFP cDNA; WPRE, posttranscriptional cis-acting regulatory element of the woodchuck hepatitis virus (587 bp); LTR-SIN, self-inactivating $3^{\prime}$-LTR deleted of 400 bp in the U3 region (29); U3, untranslated 3 ' region of the LTR; and R, repeated region of the LTR.

central nervous system (16-21); (b) intrathymic (IT) injection of a nonspecific lentiviral vector results in the transduction of thymic epithelial cells as well as a low number of immature thymocytes (22); (c) early T lymphoid progenitors (ETP) in the thymus appear to sustain production of $\mathrm{T}$ lineage progeny for longer periods of time than do the common lymphoid progenitors (CLPs) found in the BM (23). Thus we reasoned that IT injection of a T cell-specific lentiviral vector encoding a gene that provides a selective advantage for transduced prothymocytes might result in the generation of functional T lymphocyte progeny with subsequent long-term immune reconstitution.

The feasibility of this approach was assessed in a murine model of ZAP-70 deficiency. ZAP-70 is a 70-kDa protein tyrosine kinase (PTK) that is recruited to the TCR following antigenic stimulation of the TCR (24). It is expressed at approximately equivalent levels in thymocytes, mature $\mathrm{T}$ cells, and NK cells (25). Its absence results in a SCID phenotype with a block in $\mathrm{T}$ cell development at the $\mathrm{CD} 4^{+} \mathrm{CD} 8^{+}$thymocyte stage (26-28). To obviate the potential obstacles/risks concerning ubiquitous expression of ZAP-70, the human WT gene was introduced into a $T$ cell-specific lentiviral vector (29). Here we demonstrate that direct IT injection of this ZAP-70-expressing lentiviral vector results in the reconstitution of polyclonal and functional $\mathrm{T}$ cells.

\section{Results}

Thymocyte development in mice injected IT with a ZAP-70-expressing lentiviral vector. In an attempt to reconstitute $T$ lineage cells via in situ gene transfer, we injected a T cell-specific lentiviral vector encoding human ZAP-70 (pT-ZAP) directly into the thymi of 8- to 12-week-old ZAP-70-deficient mice. In PT-ZAP, the ZAP-70/internal ribosome entry site/enhanced GFP (ZAP-70/IRES/eGFP) cassette is under

\section{Figure 2}

Thymocyte differentiation in $Z A P-70^{-/}$ mice following in situ injection of pT-ZAP. pT-ZAP was injected IT into 8- to 12-week-old ZAP-70-/- mice, and thymocytes were harvested from euthanized animals 7-13 weeks later. (A) Total thymocytes were stained with $C y$-conjugated $\alpha$-CD8 and APC-conjugated $\alpha$-CD4mAbs. The percentages of DP and CD4+ and $\mathrm{CD}^{+} \mathrm{SP}$ thymocytes in WT (C57BL/6), ZAP-70---, and in mice reconstituted in vivo are indicated in each dot plot. (B) The percentages of eGFP+ cells within the entire thymus as well as within the thymocyte subset wherein TCR $\beta$ was upregulated are shown. The CD4/CD8 distributions of TCR- $\beta$-upregulated thymocytes within the eGFP- population of ZAP- $70^{-/-}$and WT mice are shown. Additionally, the CD4/CD8 distributions within the eGFP- and eGFP+ TCR- $\beta$-upregulated populations in ZAP-70-1- mice injected with pT-ZAP are compared. The mouse injected IT was sacrificed at 8 weeks after treatment. Results are representative of data obtained from 6 mice.
A

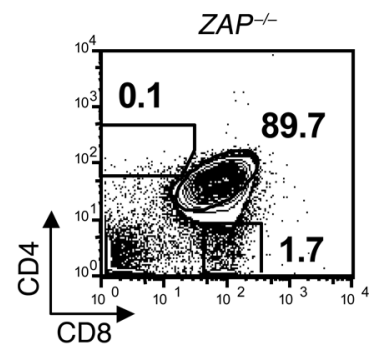

B
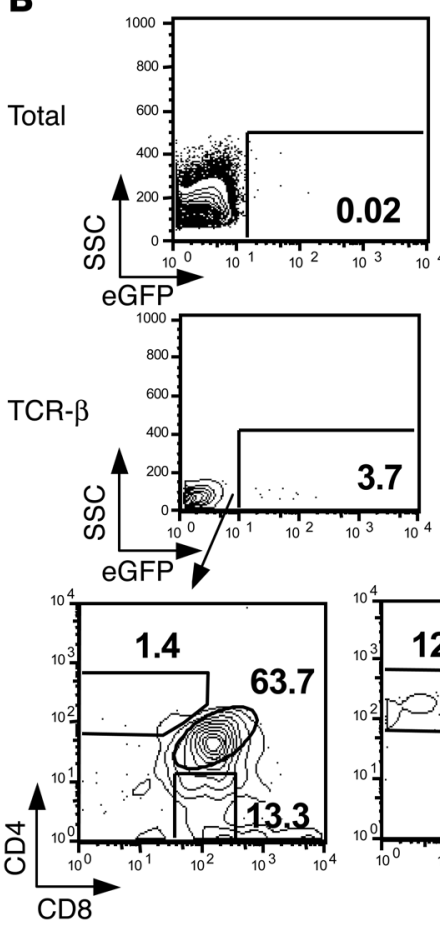
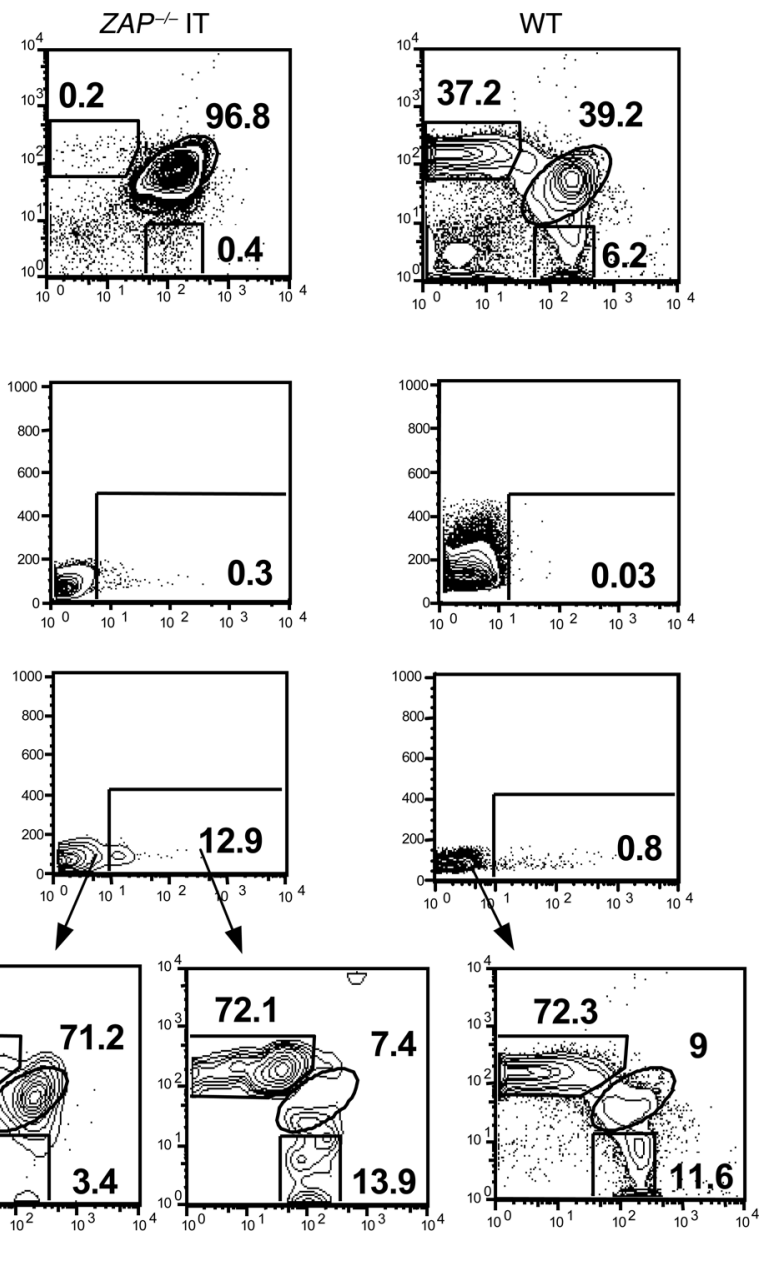


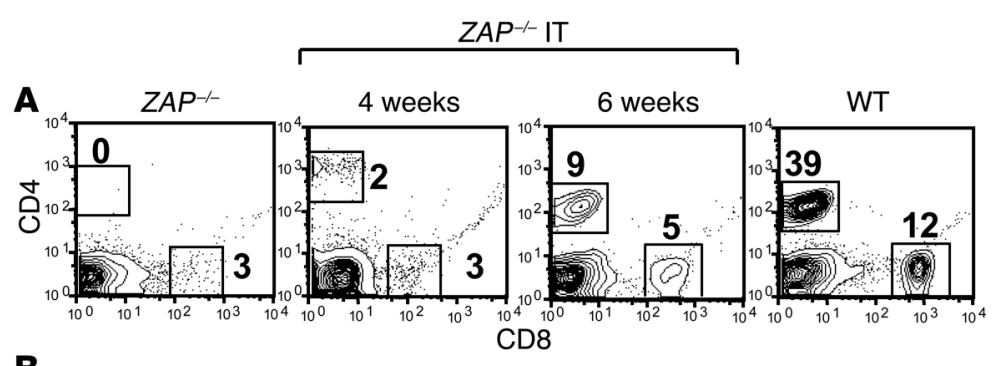

B
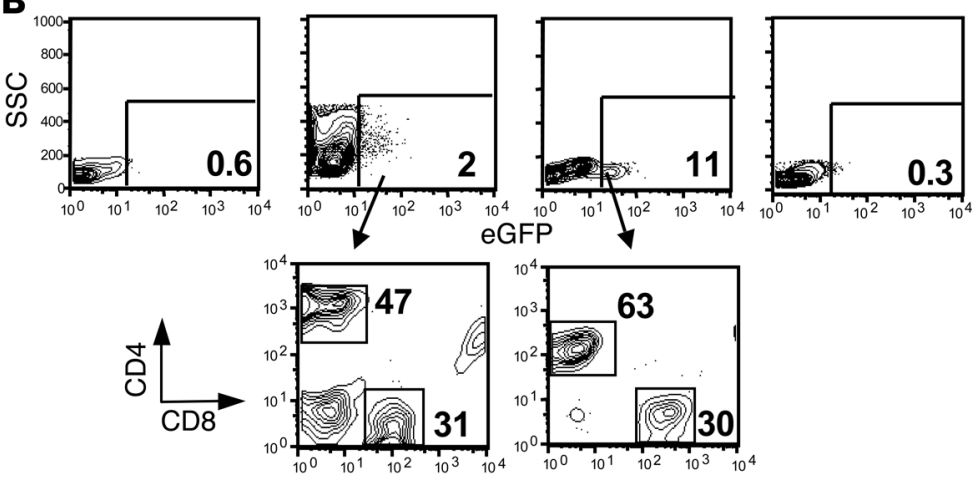

Figure 3

Kinetics of peripheral T lymphocyte emigration following in situ thymic injection of a ZAP-70-expressing lentiviral vector. (A) The presence of CD4+ and CD8 ${ }^{+} \mathrm{T}$ lymphocytes in the peripheral blood of $Z A P-70^{-1-}$ mice was monitored by flow cytometry at 4 and 6 weeks after IT injection of pT-ZAP $\left(Z A P^{-/-} I T\right)$. The overall percentage of $\mathrm{CD}^{+}$and $\mathrm{CD} 8^{+} \mathrm{T}$ cells within the peripheral blood at these time points is indicated. Analyses of representative $Z A P-70^{-/-}$and WT mice are also shown. (B) The percentages of eGFP ${ }^{+}$cells in the peripheral blood of all animals are shown. The CD4/CD8 phenotypes of the eGFP+ cells isolated from the pT-ZAP-injected mice at 4 and 6 weeks were evaluated by specific gating on this population.

the transcriptional control of $\mathrm{T}$ cell-specific regulatory sequences derived from the CD4 gene (Figure 1). In the context of this lentiviral vector, ZAP-70 and eGFP expression are concordant (Supplemental Figure 1; supplemental material available online with this article; doi:10.1172/JCI23966DS1). After thoracic surgery, we injected IT $10-30 \mu \mathrm{l}$ of vector preparations containing $2-4 \times 10^{7}$ transduction units (TUs) of pT-ZAP. It is notable that this translates into a very low overall MOI $(<0.1)$, as determined by the ratio of infectious particles to the total overall number of thymocytes and stromal cells in the thymus. Furthermore, since we have previously shown that IT injections lead to a preferential infection of thymic stromal cells, the MOI for thymocytes is even lower (22).

The absence of ZAP-70 is associated with a relatively late block in $\mathrm{T}$ cell differentiation at the $\mathrm{CD} 4^{+} \mathrm{CD}^{+}$double positive (DP) thymocyte stage (26-28) (Figure 2A). As expected from the very low MOI used, the actual percentage of transduced thymocytes was extremely low (0.3\%; Figure $2 \mathrm{~B}$ ), and thus IT injections of PT-ZAP were not expected to dramatically alter the overall percentage of single positive (SP) thymocytes as compared with the overall percentage of SP cells in deficient animals (Figure 2A). However, while TCR- $\beta$ upregulation is blocked in ZAP-70-deficient mice, IT-injected mice demonstrated a significant increase in TCR- $\beta^{+}$CD 4 SP thymocytes (data not shown). Moreover, analysis of eGFP ${ }^{+}$TCR $-\beta^{+}$ cells showed that percentages of mature CD4 and CD8 SP thymocytes were similar to those detected in WT mice, representing more than $80 \%$ of cells (Figure $2 \mathrm{~B}$ ). In marked contrast, the phenotype of thymocytes within the eGFP-TCR- $\beta^{+}$population of
pT-ZAP-injected mice was largely that of immature DP cells (71\%, Figure 2B). These results indicate that ZAP-70 expression driven from $\mathrm{PT}$-ZAP is capable of correcting the differentiation block in ZAP-70 deficiency.

As differentiating thymocytes have a limited life span, i.e., double negative (DN) cells can differentiate and migrate across the cortex in 15 days (30), the detection of transduced thymocytes at 7-52 weeks after gene transfer strongly suggests that the ZAP-70 lentiviral vector was successfully integrated into progenitor cells (Figure 2B and Supplemental Figure 4). Notably, this modulation in thymocyte differentiation was not due to a nonspecific effect of the injected virions for the following reasons: (a) mature thymocytes were not detected 14 weeks after injection of a control eGFP lentiviral vector (Supplemental Figure 2); and (b) neither injection of eGFP nor of ZAP-70 lentiviral vectors at the doses utilized here modulated thymocyte differentiation immediately (at 72 hours) following IT injection (Supplemental Figure 3).

Tcell development in mice injected IT with a ZAP-70-expressing lentiviral vector. To determine whether in vivo gene transfer in the thymus results in the appearance of mature $T$ cells in the periphery, peripheral blood samples were analyzed for the presence of $\mathrm{CD}^{+}$lymphocytes starting at 4 weeks after thymic injection (Figure 3A). We could indeed detect such cells in 6 of 26 treated adult mice. The number of $\mathrm{CD}^{+}$peripheral blood lymphocytes at 4 weeks reached $4-5 \%$, similar to what we previously observed following transplantation of WT progenitor cells or $Z A P-70^{-1-}$ progenitor cells transduced with a ZAP-70-expressing retroviral vector (31). Notably, in reconstituted mice, the percentage of peripheral blood $\mathrm{CD}^{+}$lymphocytes increased with time, with $15 \% \mathrm{CD}^{+}$or $\mathrm{CD}^{+} \mathrm{T}$ lymphocytes in the peripheral blood at 6 weeks (Figure 3A). These levels are nonetheless significantly lower than that detected in WT mice. As shown in Figure $3 \mathrm{~B}$, the vast majority of cells within the $\mathrm{GFP}^{+}$compartment were of the $\mathrm{CD}^{+}$or $\mathrm{CD}^{+} \mathrm{T}$ cell phenotype. The increment in $\mathrm{T}$ cell numbers continued in the IT-injected mice with higher percentages of Tlymphocytes detected in the lymph nodes and spleen at 8 weeks after IT injection (Figure 4A and data not shown). Altogether, these data demonstrate that a correction in ZAP-70 expression by IT gene transfer results in the presence of mature T cells in the periphery.

The activation status of these $\mathrm{T}$ lymphocytes was determined by assessing the expression of the CD25 and CD69 activation markers. $\mathrm{T}$ cells from $Z A P-70^{-/-}$mice injected IT with $\mathrm{pT}-\mathrm{ZAP}$ expressed the CD25 activation marker on a significantly higher proportion of cells than that observed in WT mice $(44 \% \pm 11.7 \%[n=5]$ vs. $22 \% \pm 4.6 \%$ $[n=5], P=0.001$; Figure 4B). Expression of the CD69 activation marker was also elevated in ZAP-70-transduced T cells as compared with T cells in WT mice, with the elevated expression being indicative of $\mathrm{T}$ cell activation. While both naive and memory phenotype (32) T cell populations were observed in reconstituted mice, the percentage of naive T cells $\left(\mathrm{CD} 6 \mathrm{~L}^{+}\right)$was lower in mice injected IT with pT-ZAP (Figure 4B). Indeed, the overall percentage of peripheral $\mathrm{T}$ cells detected in PT-ZAP-injected mice was always lower than that observed in WT animals (Table 1). CD3 ${ }^{+}$lymph node T cells in the PT-ZAP-injected mice reached $26 \%$ whereas those in WT mice averaged approximately $60 \%$ (Table 1 ). Of note, the level of eGFP expression in the $\mathrm{CD}^{+} \mathrm{T}$ cells varied widely, from 14 to $66 \%$. As $\mathrm{T}$ cell

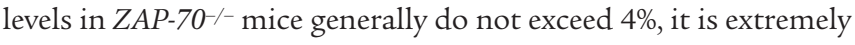



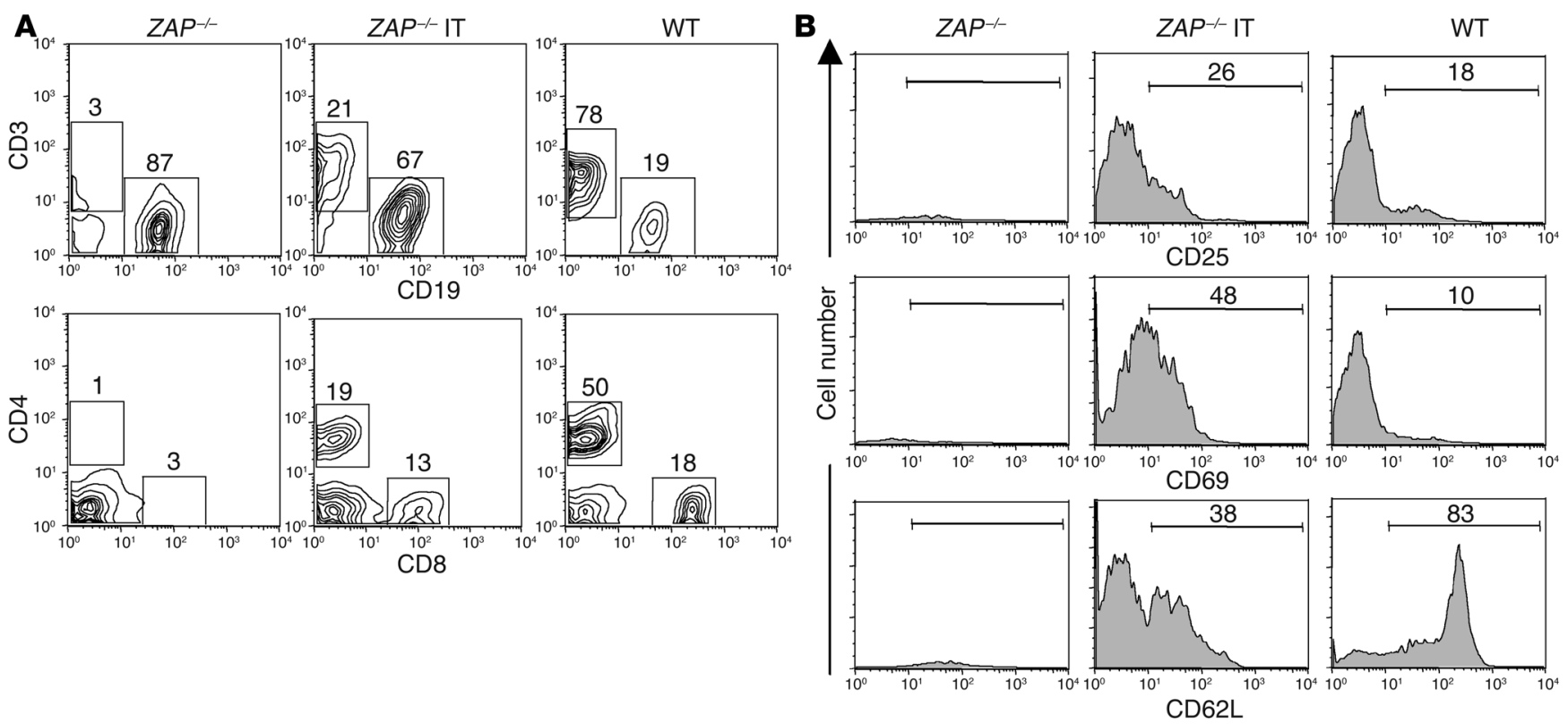

Figure 4

T lymphocyte reconstitution in ZAP-70-- mice following in situ injection of a ZAP-70-expressing lentiviral vector. Lymph nodes were collected from

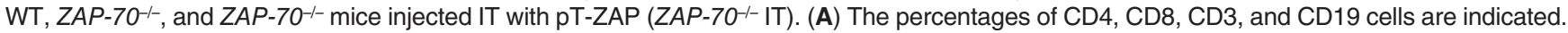
(B) The activation status of the T cell population was determined using PE-conjugated $\alpha$-CD25 and $\alpha$-CD69 mAbs, and the relative percentages of naive and memory $T$ cells were monitored with a mAb recognizing CD62L (CD62L ${ }^{+}$and CD62L-' respectively). The percentages of positively stained cells are indicated in each histogram. The representative IT-reconstituted mouse presented here was sacrificed at 8 weeks after injection.

likely that the vast majority of $\mathrm{T}$ cells detected in the PT-ZAPinjected mice differentiated due to the presence of the ZAP-70 transgene. Thus, our inability to detect eGFP in all T cells is either due to some discordance between ZAP-70 and eGFP expression in vivo in the context of the upstream IRES (Figure 1 and Table 1) or to low downregulated levels of eGFP not detected by FACS.

ZAP-70 expression and diversity of T cells that developed in mice injected IT with a ZAP-70-expressing lentiviral vector. We next assessed the level of ZAP-70 expressed in peripheral T lymphocytes as well as the diversity of their TCR repertoire (Figure 5). Levels of lentiviral-encoded ZAP-70 were similar to those of the endogenous protein in WT mice. The ectopic expression of the ZAP-70 transgene was further studied by sorting $\mathrm{CD}^{+}$cells and $\mathrm{CD}^{-}$cells from WT mice and $Z A P-70^{-/-}$mice reconstituted by IT injection of PT-ZAP. Importantly, ectopic ZAP-70 expression was detected almost exclusively in the $\mathrm{CD}^{+} \mathrm{T}$ cell compartment upon expression from the pT-ZAP lentiviral vector (Figure 5A). Thus, the CD4 promoter appears to drive expression of ZAP-70 in $\mathrm{T}$ cells to levels similar to that of the endogenous promoter.

We then investigated the relative usage of each TCR $\beta$ chain hypervariable region (TCRBV) within the global T cell population by flow cytometry and the Immunoscope/spectratype method $(33,34)$. Using this combination of methods, we established both the frequencies of $\beta$ chain hypervariable region-expressing (BV-expressing) cells and their repertoire diversity. The Immunoscope method is based on an RT-PCR of the hypervariable complementarity determining region 3 (CDR3), which allows the lengths of the mRNA encoding each $\beta$ chain of the TCR to be analyzed. In a normal setting, each single peak is composed of multiple differently rearranged sequences. A Gaussian distribution of the CDR3 lengths is thus indicative of an extremely diverse and nonbiased $\mathrm{T}$ cell population.
The CDR3 length profiles of T cells from the $Z A P-70^{-/-}$mice reconstituted by IT injection had a Gaussian distribution for an average of $26 \%$ of the $14 \mathrm{BV}$ families analyzed (with a range of 0 to $75 \%$ ), with the remaining BV families appearing as monoclonal or oligoclonal. This is in contrast with WT mice, in which a Gaussian distribution was found for all the TCRBV families analyzed (Figure 5B). Flow cytometry analyses of TCRBV expression in 6 reconstituted mice also showed a diverse, though not normal, repertoire in both CD4 and CD8 T cell populations, with BV representation varying for each $Z A P-70^{-1-}$ mouse reconstituted by IT injection. We found that, on average, $60 \%$ of the $11 \mathrm{BV}$ families analyzed were detected by flow cytometry using BV-specific antibodies (with a range of $30 \%$ to $100 \%$ ) and $77 \%$ by the Immunoscope method (with a range of $50 \%$ to $100 \%$ ). This difference is likely due to the higher sensitivity of the Immunoscope method as compared with flow cytometry. Our results thus show that the T cell repertoire of PT-ZAP-injected mice was fairly diverse in terms of BV usage but was perturbed with respect to both frequencies and clonality.

Function of T cells that developed in mice injected IT with a ZAP-70expressing lentiviral vector. The functionality of the Tlymphocytes that developed in IT-injected $Z A P-70^{-/-}$mice was assessed in several ways, including by their ability to respond to ex vivo TCR stimulation (Figure 6A). Notably, ZAP-70 plays a critical role in the activation of mature T lymphocytes. In the absence of the WT protein, early as well as late biological responses such as proliferation are defective (35). In the absence of stimulation, neither WT nor pT-ZAP-transduced T cells significantly proliferated, showing that ectopic expression of ZAP-70 did not modulate the basal proliferative status of corrected T cells. Following 3 days of stimulation with either concanavalin A or $\alpha$-CD3/IL-2, a significant percentage of both WT and PT-ZAPtransduced $\mathrm{T}$ cells had undergone up to 4 divisions. In contrast, 


\section{Table 1}

Percentages of $\mathrm{CD}^{+}$lymphocytes in $Z A P-70^{-/}$mice following in situ thymic injection of a pT-ZAP

\begin{tabular}{|c|c|c|c|c|}
\hline Mice & $\begin{array}{c}\text { Age at } \\
\text { injection } \\
\text { (weeks) }\end{array}$ & $\begin{array}{c}\text { Time } \\
\text { after } \\
\text { injection } \\
\text { (weeks) }\end{array}$ & $\begin{array}{c}\text { CD3 } \\
\text { T cells } \\
(\%)\end{array}$ & $\begin{array}{c}\text { eGFP+ } \\
\text { T cells } \\
(\%)\end{array}$ \\
\hline$Z A P^{-/-1}$ & / & / & 3 & 1 \\
\hline$Z A P^{-/-2}$ & / & I & 4 & 2 \\
\hline$Z A P^{-1-} 3$ & / & l & 3 & $<0.5$ \\
\hline$Z A P^{-1}-1 \mathrm{~T} 1$ & 12 & 20 & 23 & 14 \\
\hline$Z A P^{-/-}$IT 2 & 12 & 52 & 19 & 44 \\
\hline$Z A P^{-1-}$ IT 3 & 9 & 8 & 26 & 66 \\
\hline$Z A P^{-/-} \mathrm{IT} 4$ & 9 & 10 & 13 & 35 \\
\hline$Z A P^{--}$IT 5 & 4 & 20 & 23 & 25 \\
\hline$Z A P^{-1-}$ IT 6 & 2 & 20 & 11 & 15 \\
\hline$Z A P^{-1-} \mathrm{IT} 7$ & 2 & 20 & 20 & 25 \\
\hline$Z A P^{-1-} \mid \mathrm{IT} 8$ & 2 & 20 & 16 & 41 \\
\hline$Z A P^{-/} \mid \mathrm{IT} 9$ & 2 & 15 & 11 & 48 \\
\hline$Z A P^{-/-}$IT 10 & 2 & 17 & 16 & 42 \\
\hline$Z A P^{-/}-1 \mathrm{~T} 11$ & 2 & 17 & 10 & 14 \\
\hline WT 1 & / & l & 75 & $<0.5$ \\
\hline WT 2 & / & / & 51 & $<0.5$ \\
\hline WT 3 & / & / & 64 & $<0.5$ \\
\hline
\end{tabular}

ZAP-70-/- mice were injected with the ZAP-70-expressing lentiviral vector at the indicated ages and analyzed at 8-52 weeks after injection as noted. The percentages of CD3+ T cells in the lymph nodes of sacrificed animals as well as the percentages of eGFP+ cells within the $\mathrm{CD}^{+}$ populations were determined by flow cytometry. Note that presented analyse of IT-injected mice are limited to those mice showing T cell reconstitution for whom lymph node analyses were performed. Slashes indicate control mice that were not injected. The percentages of $\mathrm{T}$ cells in control ZAP-70-/- and WT mice are shown.

no division was induced in splenocytes isolated from control ZAP-70deficient mice. Thus, pT-ZAP-transduced T cells, like WT T cells, are capable of responding to a TCR-specific stimulus.

To assess whether these pT-ZAP-transduced T cells were capable of responding to immune stimulation in vivo, mice were grafted with both syngeneic (C57BL/6) and fully mismatched allogeneic $(\mathrm{BALB} / \mathrm{c})$ skin grafts on the 2 sides of the back. Control mice included untreated ZAP-70-deficient mice $(n=3)$ and WT C57BL/6 mice $(n=2)$ transplanted with the same skin grafts. As expected, the untreated ZAP-70-deficient mice, who do not develop T cells, rejected neither the syngeneic nor the allogeneic grafts. In the control C57BL/6 mice, allograft rejection was acute, with signs of necrosis appearing from day 12 onward while syngeneic grafts were maintained. In the pT-ZAP-injected mice, signs of rejection were observed in 2 of the 3 mice between days 21 and 30. Importantly, the 2 mice who presented with erythema and thickening of the allogeneic graft had higher percentages of $\mathrm{CD}^{+}$peripheral $\mathrm{T}$ cells (16\% and $19 \%$ splenic T cells, respectively) than the mouse without evidence of rejection (7\%). So the grafts could be more precisely analyzed, they were biopsied between days 30-40, and histologi$\mathrm{cal}$ analyses were performed on paraffin sections. In the syngeneic and allogeneic sections transplanted on the pT-ZAP-injected mice, there was evidence of fibroblast hyperplasia. However, only in allogeneic sections was lymphocyte infiltration prevalent in the superficial dermis and epidermal layers (in the 2 of 3 mice with clinical evidence of rejection; Figure 6B). Moreover, the T lymphocytes from the IT-injected mice proliferated in response to these alloantigens, as observed in a mixed lymphocyte reaction. Lymph node $\mathrm{T}$ cells from WT (C57BL/6) and skin-grafted pT-ZAP-reconstituted mice proliferated in response to allogeneic BALB/c antigens (Figure $6 \mathrm{C}$ ). Thus, allogeneic immune stimulation of PT-ZAP-transduced $\mathrm{T}$ cells was induced by the presence of a mismatched skin graft.

$T$ cell reconstitution efficiency is significantly enhanced upon IT injection of a ZAP-70-expressing lentiviral vector into infant mice. Taken together, the data presented above demonstrate that IT injection of a pT-ZAP can result in the restoration of T cell differentiation in the thymus and periphery of ZAP-70-deficient mice. Nevertheless, we were only able to detect significant T cell reconstitution in 6 of 26 adult $Z A P-70^{-/-}$mice injected IT with pT-ZAP (23\%). We believe that this relatively low efficacy is in large part due to (a) technical issues related to injection of the virion suspension into the small thymus of adult mice and (b) the low MOI used. Moreover, insufficient ZAP-70 transgene expression in the thymus and/or an insufficient population of thymocyte progenitors that are susceptible to lentiviral transduction may also play a role.

To attempt to circumvent these problems, we injected pT-ZAP into the thymi of young mice (10-14 days). As in human infants, the thymi of ZAP-70-deficient infant mice are significantly larger proportionately than those of adults. Moreover, we found that a higher percentage of prothymocytes in the thymi of ZAP-70-deficient infants were "blast-like" as assessed by their forward angle scatter (FSC) and side angle scatter (SSC) profiles and in cycle $\left(\mathrm{Ki}^{+}\right)$as compared with their adult counterparts (Figure 7A). As we and others have shown that cycling $T$ cells are significantly more susceptible to lentiviral transduction than the quiescent population (36), prothymocytes of young mice would also be expected to be more susceptible to gene transfer. In line with this hypothesis, we found that a higher percentage of the $\mathrm{Ki}^{+} 7^{+}$thymocytes in infants were of an immature DN phenotype (25\% vs. $14 \%$; Figure $7 \mathrm{~B})$.

Since performing thoracic surgery on 10- to 14-day-old mice is technically challenging, we developed a blind approach whereby the virion suspension was injected directly through the skin into the thoracic cavity immediately above the sternum. This approach resulted in appropriately targeted IT injection, as assessed using injected dyes (our unpublished observations). Following IT injection of PT-ZAP into these infant thymi, $\mathrm{T}$ cell reconstitution was detected in $75 \%$ of injected animals (15 of 20), with percentages of $\mathrm{CD}^{+} \mathrm{T}$ cells in lymph nodes and spleens similar to that observed in successfully treated adult mice (Figure $7 \mathrm{C}$ and Table 1). Thus, direct in vivo injection of ZAP-70-expressing lentiviral vectors into infant thymi results in a significantly improved success rate of $\mathrm{T}$ cell reconstitution.

\section{Discussion}

Altogether, our data demonstrate that direct injection of $\mathrm{T}$ cellspecific lentiviral vectors into thymi of ZAP-70-deficient mice allows long-term $\mathrm{T}$ cell reconstitution and transgene expression in peripheral $\mathrm{T}$ lymphocytes. The efficacy of this treatment is in large part due to the context of SCID where the transgene confers a strong selective advantage (4-7). Importantly, this selective advantage is fully exploited by IT injection; we failed to observe $T$ cell reconstitution upon i.v. injection of the pT-ZAP vector $(\mathrm{O}$. Adjali, M. Steinberg, and N. Taylor, unpublished observations).

Transduction of early Tcell progenitors by ITlentiviral vectorinjections. Upon IT gene transfer, it was possible that the peripheral $T$ lymphocytes in pT-ZAP-treated mice arose from the expansion of a clonal or oligoclonal population of mature thymocytes derived from DP thymocytes 
A

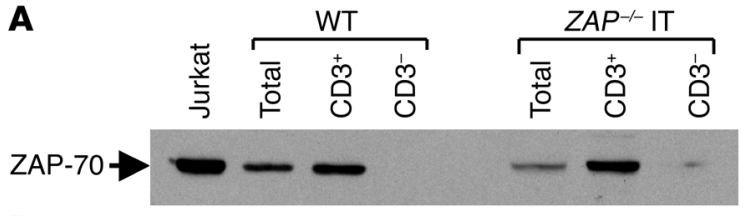

B
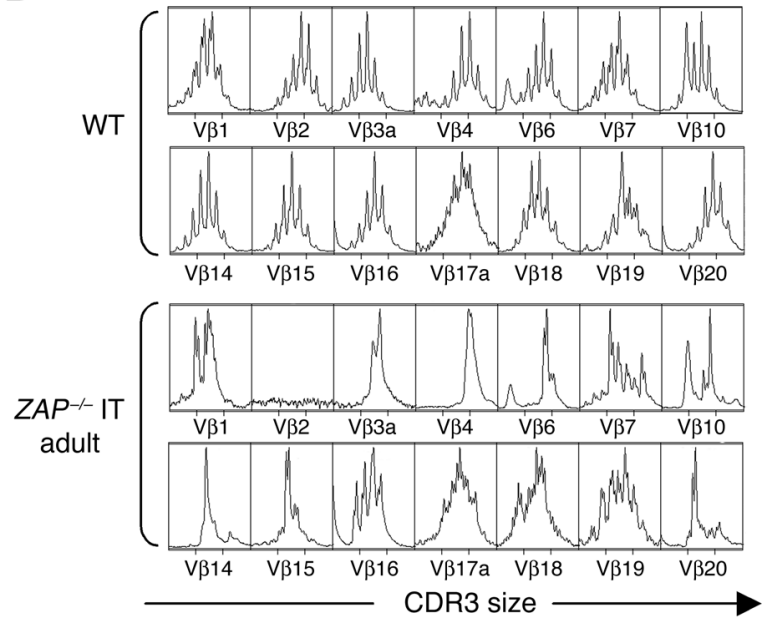

transduced after $\beta$ chain rearrangement. Our results argue against this hypothesis. Indeed, we previously reported that transduced DN cells, which contain the immature $\mathrm{T}$ cell progenitors, can be detected shortly after IT lentiviral injections of a nonspecific (21) or a $\mathrm{T}$ cell-specific lentiviral vector (our unpublished observations). Moreover, gene-modified thymocytes were detected for significant time periods, albeit at small numbers and in a low percentage of animals (<25\%). In 1 pT-ZAP-injected animal who was maintained for 1 year, immature DN and DP thymocytes expressing eGFP (0.5\%) were still detected at 1 year (Supplemental Figure 4). Nevertheless, it is not clear whether this is due to the maintenance of progenitor cells in the thymus or, more likely, to the recirculation of transduced progenitors into the thymus. The second hypothesis is supported by our finding that transduced lineage-negative progenitor cells can be detected in the bone marrow at more than 8 weeks following injection of pT-ZAP into the thymus (our unpublished observations). Thus, it will be important to assess the fate of thymic progenitors and determine whether a subpopulation of these cells can emigrate from the thymus and then reenter at later time points.

$T$ cell diversity and function. The Immunoscope method is based on an RT-PCR of the hypervariable CDR3 and allows the lengths of the mRNA encoding each $\beta$ chain of the TCR to be analyzed. In a normal setting, each peak is composed of multiple differently rearranged sequences. The detection of single peaks for some BV families is indicative of the expansion of single clones that would be preferentially amplified by PCR, and as such, may, by competing for PCR primers, prevent the detection of other CDR3 products that may nevertheless be present.

The level and diversity of the PT-ZAP-reconstituted peripheral $\mathrm{T}$ cells are sufficient for providing ZAP-70 immunodeficient mice with functional $\mathrm{T}$ cells that respond to mitogenic stimulation in vitro and mount in vivo immune responses to skin allografts. Upon improvement of the methodology, it will be important to assess the immune responses to nominal antigen and/or infectious challenges. However, it is important to note that the TCRBV

\section{Figure 5}

ZAP-70 expression and diversity in T lymphocytes reconstituted by IT injection of pT-ZAP. (A) ZAP-70 levels in total splenocytes from WT and IT-reconstituted ZAP-70 mice as well as in sorted CD3 $3^{+}$and CD3- populations were assessed by Western blot using an $\alpha$ ZAP-70 mAb. (B) The TCRBV repertoire was assessed by a comparison of TCR CDR3 size distribution (Immunoscope profiles) of lymph node cells obtained from WT and IT-reconstituted adult $Z A P-70^{-/-}$mice. PCR products were generated by reverse transcription with different TCRBV subfamily-specific primers and $1 \mathrm{C} \beta$ primer; this was followed by a runoff reaction with a fluorescent $C \beta$ primer. The histograms represent fluorescence intensity in arbitrary units ( $y$ axis) plotted against CDR3 size ( $x$ axis). Representative results showing the size distributions within 14 TCRBV families are shown.

profiles detected in the IT-treated $Z A P^{-/-}$animals is reminiscent of that of an X-SCID patient whose T cells developed from a reverse mutation in a single $T$ cell precursor (9), resulting in a fairly positive clinical condition (37). Thus, the rescue of few progenitor/ stem cells can promote the differentiation of $\mathrm{T}$ cells with a partially diversified TCR repertoire, sufficient to provide individuals with protection from infection and a positive clinical outcome.

Potential safety advantages of in situ gene therapy for SCID. It is noteworthy that in mice injected with the vector utilized here wherein eGFP was driven from a minimal CD4 promoter, eGFP expression was not detected in thymic stroma or liver, which were the main targets transduced following IT injection using ubiquitous lentiviral vectors (22). In general, this is a major advantage of lentiviral vectors, wherein lineage specificity can be obtained and contributes to the overall safety of the approach. Another advantage of our approach is that gene transfer was obtained in the absence of prior conditioning. Indeed, the vast majority of studies transplanting gene-modified HSC into murine SCID models were performed in lethally irradiated mice $(31,38,39)$. Moreover, in human patients with ADA deficiency, a high level of immune reconstitution following injection of ex vivo gene-corrected HSC has been shown to require pretransplantation chemotherapy (6). Importantly, in ZAP-70deficient mice, $T$ cell reconstitution by HSC corrected ex vivo with a $\mathrm{ZAP}-70$ retroviral vector does not occur in the absence of conditioning (O. Adjali, M. Steinberg, and N. Taylor, unpublished observations). Safety of the IT gene transfer protocol in terms of potential insertional mutagenesis or autoimmunity remains to be fully investigated but is likely to be diminished by the low MOI used in the in situ injections. Altogether though, we believe that the IT injection of T cell-specific lentiviral vectors without preconditioning represents a safety improvement for SCID gene therapy.

Perspectives. We show that a higher frequency of reconstitution was obtained in infant as compared with adult mice, further highlighting the potential of our approach in ZAP-70-deficient children. In view of a potential clinical application, we recently assessed this approach in macaques, performing direct IT injection under ultrasound guidance (P. Moullier [INSERM, Nantes, France], personal communication) and determined it to be a simple and feasible technical gesture. We believe that the overall efficacy of our approach will be significantly improved in a clinical setting. Indeed, IT gene transfer of lentiviral vectors is not optimal when applied to mice for the following reasons: (a) at similar MOIs and at equivalent levels of activation, murine $\mathrm{T}$ cells are less susceptible to lentiviral infection than are human $\mathrm{T}$ cells (our unpublished observations and ref. 36); and (b) the small size of the mouse thymus allows neither the injec- 
A

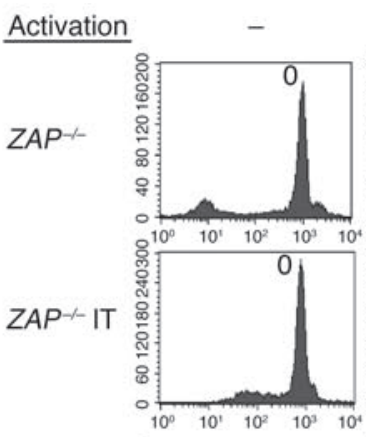

Con A

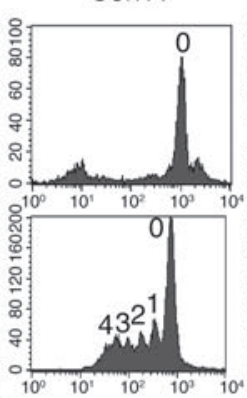

WT

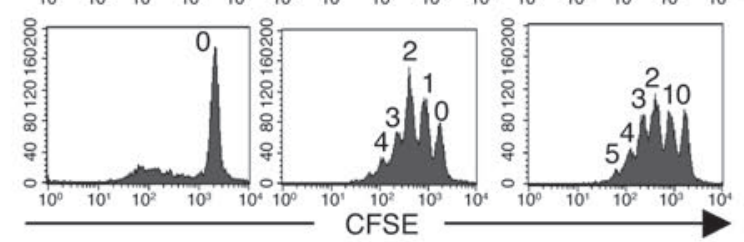

$\alpha \mathrm{CD} 3 / \mathrm{IL}-2$

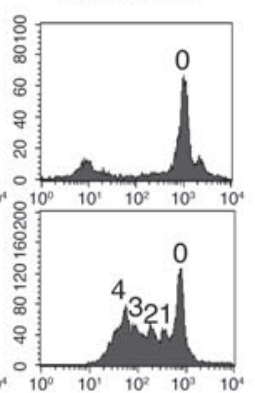

C

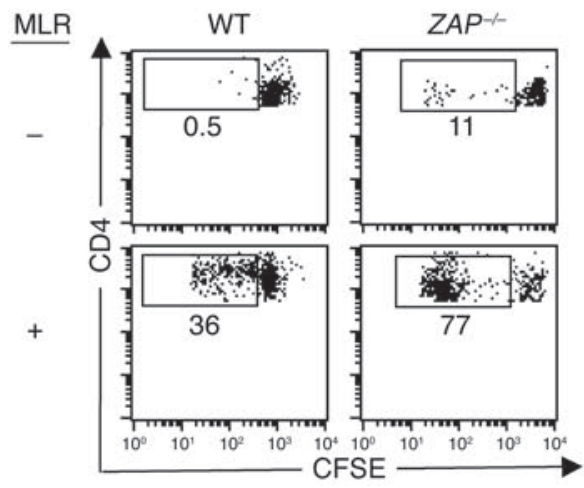

B

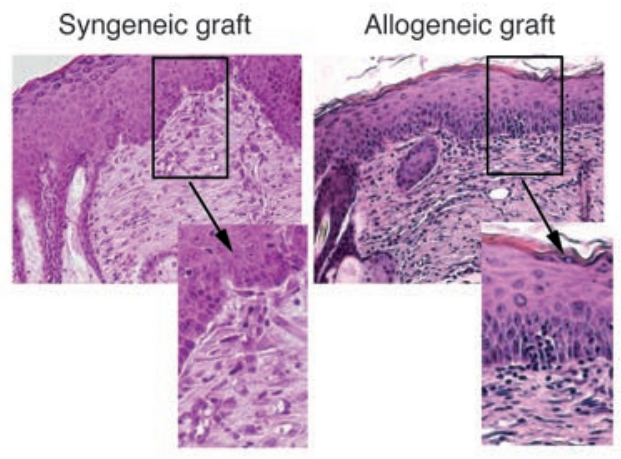

\section{Figure 6}

In vitro and in vivo responsiveness of T lymphocytes reconstituted by IT injection of pT-ZAP. (A) Splenocytes from ZAP-70-/-, WT, and IT-reconstituted $Z A P-70^{-/-}$mice were labeled with the fluorescent dye CFSE and cultured in vitro in the presence of concanavalin A (Con A) or $\alpha \mathrm{CD} 3 / \mathrm{IL}-2$ for 3 days. Cells were analyzed for CFSE intensity by flow cytometry. The numbers shown above the peaks indicate the number of cell divisions. The IT-reconstituted ZAP-70 mice were sacrificed at 8 weeks after injection. (B) Histological sections of syngeneic (C57BL/6) and allogeneic (BALB/c) skin sections, which had both been grafted on the same IT-reconstituted ZAP-70 mouse, were stained with H\&E. The presence of lymphocytes (shown as dark purple staining) infiltrating into the epidermis is shown in the enlarged inset. The IT-reconstituted mouse was injected 14 weeks prior to skin grafting, and skin histology was performed 30 days later. Magnification, $\times 200$. (C) Lymph node cells from WT (C57BL/6) and IT-reconstituted ZAP-70 mice (pregrafted as described above; sacrificed at 6 weeks after graft) were labeled with CFSE and cultured in the absence or presence of allogeneic BALB/c splenocytes. After 3 days, cells were stained with an $\alpha C D 4$ mAb, and the percentages of CD4 ${ }^{+}$cells that divided, as assessed by a loss of CFSE intensity, are indicated in each dot plot. MLR, mixed lymphocyte reaction.

tion of large quantities of vectors nor repeat injections. Likewise, while the translation of gene therapy from mice to larger animals or even humans has generally resulted in decreased efficiencies, we believe that this would not be the case in this setting. Thus, gene transfer using T cell-specific lentiviral vector calls for further development of this strategy for the treatment of SCID.

\section{Methods}

Lentiviral construction and virus production. The plasmid pGM1 encoding for a T cell-specific lentiviral vector expressing eGFP has been previously described (29). To obtain a T cell-specific lentiviral vector encoding ZAP-70 and eGFP, a blunted-BamHI/SalI ZAP-70/ IRES/eGFP insert from plasmid LZRS pBMN ZAP-70/IRES/eGFP (40) was inserted between EcoRV-SalI of the pGM1 plasmid (29) in replacement of the original eGFP coding sequences. The ensuing T-cell-specific lentiviral vector $\mathrm{pRRLSIN/cPPT/CD4pmE/}$ ZAP-70/IRES/eGFP/WPRE is hereafter referred to as pT-ZAP.
Virions were produced by transient calcium phosphate cotransfection of $293 \mathrm{~T}$ cells with the vector plasmid, an encapsidation plasmid lacking Vif, Vpr, Vpu, and Nef accessory HIV-1 proteins (p8.91), and a vesicular stomatitis virus-G protein (VSV-G) envelope expression plasmid ( $\mathrm{pHCMV}-\mathrm{G}$ ) (41). Viral titers, expressed as TUs, were determined by assessing transductions of Jurkat $T$ cells with serial dilutions of virion preparations. Expression of ZAP-70 and eGFP from this cassette is proportional as assessed in vitro using the Jurkat $\mathrm{T}$ cell line (data not shown).

Mice and IT injections. ZAP-70-/- mice, kindly provided by A. Singer and R. Bosselut (NIH), were bred and maintained under pathogen-free conditions. IT injections were performed at either 2-3 weeks of age (infant mice) or 8-12 weeks of age (adult mice). In the former case, virions in a total volume of 10-30 $\mu 1$ were injected directly through the skin into the thoracic cavity immediately above the sternum, using a 0.3 - $\mathrm{ml}$, 28-gauge, $8-\mathrm{mm}$ insulin syringe. Adult mice were anesthetized with $40 \mathrm{mg} / \mathrm{kg}$ of 


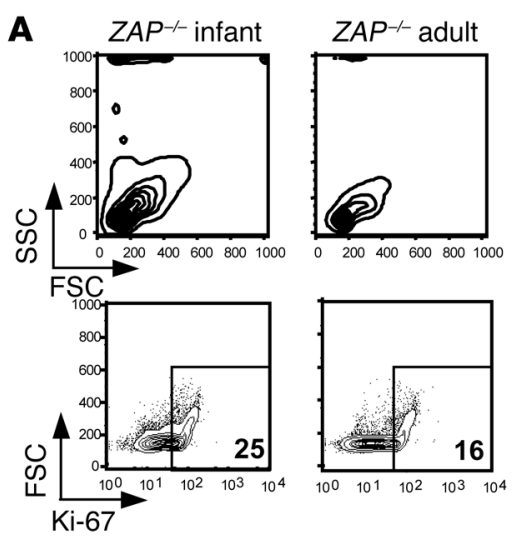

B

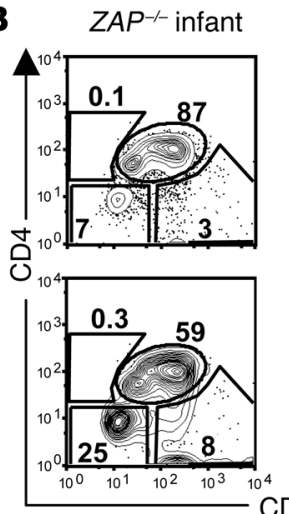

$Z A P^{-/-}$adult
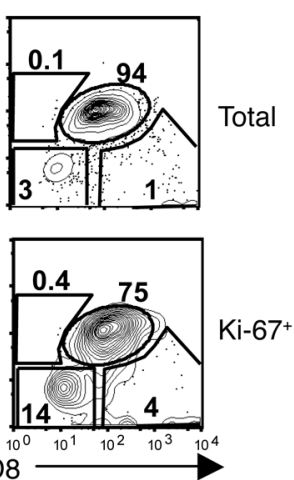

C

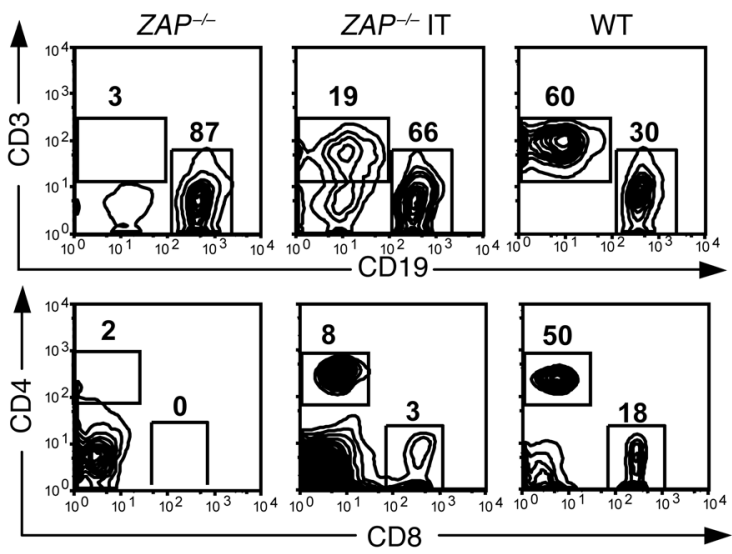

Figure 7

Enhanced T cell reconstitution efficacy following IT injection of a pT-ZAP in infant mice. (A) The FSC and SSC profiles of thymi from a 2-week-old (infant) and adult ZAP-70 KO mouse are shown. (B) Cell cycle entry of total thymocytes from an infant and adult ZAP-70 KO mouse was monitored by assessing intracellular expression of the Ki67 antigen using a fluorochrome-conjugated mAb. The CD4/CD8 distribution within the fractions of Ki67+ thymocytes is shown. (C) $\mathrm{T}$ cell reconstitution in the lymph node of a ZAP-70 KO infant mouse injected IT with pT-ZAP was monitored by flow cytometry and is representative of 15 of 20 IT-treated infant mice.
Pro version 4.0.2 (BD) or FlowJo software version 4.6.1 (Tree Star Inc.).

Skin transplantation. Skin grafts were performed on anesthetized WT (C57BL/6) and ZAP-70-deficient mice as well as on ZAP-70deficient mice with reconstituted $T$ cells 3 months after IT injection of PT-ZAP. Full-thickness skin grafts $\left(0.5 \mathrm{~cm}^{2}\right)$ were prepared from the base of both syngeneic C57BL/6 and allogeneic BALB/c donor tails. Graft beds were prepared on the right and left lateral backs of recipient mice. Skin grafts from both C57BL/6 and $\mathrm{BALB} / \mathrm{c}$ mice were attached to the backs of each mouse with interrupted sutures of silk thread, and graft appearance was monitored 3 times a week. Paraffin sections were prepared following formalin fixation of biopsied grafts. Sections were stained with $\mathrm{H} \& \mathrm{E}$.

TCR CDR3 size analyses. Total RNA was prepared using RNAble (Eurobio). Five $\mu \mathrm{g}$ of RNA was reverse transcribed using oligonucleotide $(\mathrm{dT})$ primers (Pharmacia Biotech) and Moloney-murine leukemia virus (M-MLV) reverse transcriptase (Invitrogen Corp.). cDNAs were amplified (cycles: 1 minute at $94^{\circ} \mathrm{C}, 1$ minute at $60^{\circ} \mathrm{C}$, and 2 minutes at $72^{\circ} \mathrm{C}$ ) using 2.5 IU of the AmpliTaq polymerase (Roche Diagnostics Corp.) in a 50- $\mu$ l reaction mixture with 1 of 14 TCRBV subfamily-specific primers and a $C \beta$ primer that recognizes the constant regions $C \beta 1$ and $C \beta 2$ of the $\beta$ chain of the TCR. Primers are described in refs. 43 and 44, except for V $\beta 3$ (GCAAAGATGAGGTGTATCCCTG) and C $\beta 4$ (GCCCATGGAACTGCACTTGGC) primers (Proligo pentobarbital. Midincision of the lower neck was performed to gain access to the trachea. Incision of the sternum was performed on the first 2 ribs, and virions were injected into the visualized thymus. All experiments were approved by the Institutional Review Board at the Animal Facility of the Institut de Génétique Moléculaire de Montpellier.

Immunophenotyping, proliferation, and flow cytometry analyses. ZAP-70 $70^{-/-}$mice were killed and dissected 6-18 weeks after injection. Controls were obtained from age-matched $129 / \mathrm{Sv}$ mice. Cells from lymph nodes, spleen, and thymus were stained with the indicated conjugated antibodies (BD Biosciences - Pharmingen). For proliferation analyses, splenocytes were resuspended at a concentration of $2.5 \times 10^{6}$ cells $/ \mathrm{ml}$ and labeled with the fluorochrome CFSE (Invitrogen Corp.) as previously described (42). Cells were cultured as indicated in RPMI supplemented with $10 \%$ FCS and $10 \mu \mathrm{M}$ $\beta$-mercaptoethanol in the presence of a soluble $\alpha$-CD3 mAb $(1 \mu \mathrm{g} / \mathrm{ml}$; BD Biosciences - Pharmingen) or concanavalin A $(1 \mu \mathrm{g} / \mathrm{ml})$. Alternatively, a mixed lymphocyte reaction was performed by culturing lymph node cells $\left(5 \times 10^{5} /\right.$ well $)$ in IL-2-supplemented media $(50 \mathrm{IU} / \mathrm{ml})$ in the presence or absence of allogeneic BALB/c splenocytes $\left(5 \times 10^{5} /\right.$ well $)$. All acquisitions were performed on a FACSCalibur (BD), and analyses were performed with CellQuest
France SAS). The C $\beta$ fluorescent probe was mC $\beta$ fl6-ROX 5'CTTGGGTGGAGTCACATTTCTC-3'. The final concentration was $0.5 \mu \mathrm{M}$ for each primer, $0.2-\mathrm{mM}$ dNTPs, and $2-\mathrm{mM} \mathrm{MgCl} 2$ in $1 \times$ PCR Buffer (Roche Diagnostics Corp.). For each PCR, $2 \mu 1$ was further processed in a run-off reaction (10 cycles: 1 minute at $94^{\circ} \mathrm{C}, 1$ minute at $60^{\circ} \mathrm{C}$, and 2 minutes at $72^{\circ} \mathrm{C}$ ) using $0.2 \mu \mathrm{M}$ of a ROX-labeled C $\beta$ primer (Proligo France SAS) and 2.5 IU of AmpliTaq polymerase (Roche Diagnostics Corp.). Each run-off product was denaturated and loaded on a gel for fluorescence analysis using an Applied Biosystems ABI Prism 377 DNA Sequencer. To obtain the CDR3 length profiles depicted in Figure 6, raw data were analyzed using Immunoscope 3.01b software (LogINSERM). The TCRBV nomenclature proposed by Arden et al. was used in this study (45).

Statistical analyses. Statistical significance was determined using a 1-tailed paired Student's $t$ test. $P$ values of less than 0.05 were considered to be statistically significant. All data are presented as means $\pm \mathrm{SD}$.

\section{Acknowledgments}

We thank N. Noraz, S. Choquart, R. Vicente, and V. Zimmermann for their critical input and assistance during the course of 
this study. We are indebted to K. Chebli for his scientific supervision of all mouse experiments. We are grateful to G. Barneon and D. Antonelli for expert assistance with histological and TCR CDR3 size analyses, respectively. O. Adjali, G. Marodon, and M. Steinberg were supported by fellowships from the French Ministry of Higher Education, Association Française contre les Myopathies (AFM) and Fondation pour la Recherche Medicale, and AFM and Fundacion YPF, respectively. This work was supported by the Immune Deficiency Foundation, the AFM, the Association France-Israel pour la Recherche en Science et Technologie, the Association pour la Recherche sur le Cancer, March of Dimes grant 6-FY99-406, the Centre National de la Recherche Scientifique, and INSERM.

1. Antoine, C., et al. 2003. Long-term survival and transplantation of haemopoietic stem cells for immunodeficiencies: report of the European experience 1968-99. Lancet. 361:553-560.

2. Buckley, R.H., et al. 1999. Hematopoietic stemcell transplantation for the treatment of severe combined immunodeficiency. N. Engl. J. Med. 340:508-516.

3. Patel, D.D., et al. 2000. Thymic function after hematopoietic stem-cell transplantation for the treatment of severe combined immunodeficiency. N. Engl. J. Med. 342:1325-1332.

4. Kohn, D.B., et al. 1998. T lymphocytes with a normal ADA gene accumulate after transplantation of transduced autologous umbilical cord blood CD34+ cells in ADA- deficient SCID neonates. Nat. Med. 4:775-780.

5. Cavazzana-Calvo, M., et al. 2000. Gene therapy of human severe combined immunodeficiency (SCID)-X1 disease. Science. 288:669-672.

6. Aiuti, A., et al. 2002. Correction of ADA-SCID by stem cell gene therapy combined with nonmyeloablative conditioning. Science. 296:2410-2413.

7. Gaspar, H.B., et al. 2004. Gene therapy of X-linked severe combined immunodeficiency by use of a pseudotyped gammaretroviral vector. Lancet. 364:2181-2187.

8. Hirschhorn, R., et al. 1996. Spontaneous in vivo reversion to normal of an inherited mutation in a patient with adenosine deaminase deficiency. Nat. Genet. 13:290-295.

9. Bousso, P., et al. 2000. Diversity, functionality, and stability of the $\mathrm{T}$ cell repertoire derived in vivo from a single human T cell precursor. Proc. Natl. Acad. Sci. U. S. A. 97:274-278.

10. Stephan, V., et al. 1996. Atypical X-linked severe combined immunodeficiency due to possible spontaneous reversion of the genetic defect in T cells. N. Engl. J. Med. 335:1563-1567.

11. Ariga, T., et al. 2001. T-cell lines from 2 patients with adenosine deaminase (ADA) deficiency showed the restoration of ADA activity resulted from the reversion of an inherited mutation. Blood. 97:2896-2899.

12. Cavazzana-Calvo, M., et al. 1999. Correction of SCID-XI disease phenotype following ge gene transfer by a retroviral vector into CD34+ cells in two children [abstract]. Blood. 94(Suppl. 1):367a.

13. Fischer, A., Hacein-Bey, S., and Cavazzana-Calvo, M. 2002. Gene therapy of severe combined immunodeficiencies. Nat. Rev. Immunol. 2:615-621.

14. Hacein-Bey-Abina, S., et al. 2003. A serious adverse event after successful gene therapy for X-linked severe combined immunodeficiency. N. Engl.J. Med. 348:255-256.

15. Li, Z., et al. 2002. Murine leukemia induced by retroviral gene marking. Science. 296:497.

16. Esslinger, C., et al. 2003. In vivo administration of a lentiviral vaccine targets DCs and induces
Received for publication November 22, 2004, and accepted in revised form May 31, 2005.

Address correspondence to: Naomi Taylor, Institut de Génétique Moléculaire de Montpellier, CNRS UMR 5535/IFR 122, 1919 route de Mende, F-34293 Montpellier Cedex 5, Paris, France. Phone: 33-467-61-36-28; Fax: 33-467-04-020-31; E-mail: taylor@ igm.cnrs-mop.fr. Or to: David Klatzmann, UPMC/CNRS UMR7087, Hôpital Pitié-Salpêtrière, 83 Bd de l'hôpital, 75651 Paris, France. Phone: 33-142-17-74-61; Fax: 33-142-17-74-62; E-mail: david.klatzmann@chups.jussieu.fr.

\section{Naomi Taylor and David Klatzmann are co-senior authors.}

efficient CD8(+) T cell responses. J. Clin. Invest. 111:1673-1681. doi:10.1172/JCI200317098.

17. VandenDriessche, T., et al. 2002. Lentiviral vectors containing the human immunodeficiency virus type- 1 central polypurine tract can efficiently transduce nondividing hepatocytes and antigenpresenting cells in vivo. Blood. 100:813-822.

18. Yanay, O., et al. 2003. Treatment of canine cyclic neutropenia by lentivirus-mediated G-CSF delivery. Blood. 102:2046-2052.

19. Palmowski, M., Salio, M., Dunbar, R.P., and Cerundolo, V. 2002. The use of HLA class I tetramers to design a vaccination strategy for melanoma patients. Immunol. Rev. 188:155-163.

20. Kobinger, G.P., Louboutin, J.P., Barton, E.R., Sweeney, H.L., and Wilson, J.M. 2003. Correction of the dystrophic phenotype by in vivo targeting of muscle progenitor cells. Hum. Gene Ther. 14:1441-1449.

21. Baekelandt, V., Eggermont, K., Michiels, M., Nuttin, B., and Debyser, Z. 2003. Optimized lentiviral vector production and purification procedure prevents immune response after transduction of mouse brain. Gene Ther. 10:1933-1940.

22. Marodon, G., and Klatzmann, D. 2004. In situ transduction of stromal cells and thymocytes upon intrathymic injection of lentiviral vectors. BMC Immunol. 5:18.

23. Allman, D., et al. 2003. Thymopoiesis independent of common lymphoid progenitors. Nat. Immunol. 4:168-174.

24. Chan, A.C., Iwashima, M., Turck, C.W., and Weiss, A. 1992. ZAP-70: a $70 \mathrm{kd}$ protein-tyrosine kinase that associates with the TCR zeta chain. Cell. 71:649-662.

25. Chan, A.C., et al. 1994. Differential expression of ZAP-70 and Syk protein tyrosine kinases, and the role of this family of protein tyrosine kinases in TCR signaling. J. Immunol. 152:4758-4766.

26. Negishi, I., et al. 1995. Essential role for ZAP-70 in both positive and negative selection of thymocytes. Nature. 376:435-438.

27. Wiest, D.L., et al. 1997. A spontaneously arising mutation in the DLAARN motif of murine ZAP-70 abrogates kinase activity and arrests thymocyte development. Immunity. 6:663-671.

28. Kadlecek, T.A., et al. 1998. Differential requirements for ZAP-70 in TCR signaling and T cell development. J. Immunol. 161:4688-4694.

29. Marodon, G., et al. 2003. Specific transgene expression in human and mouse CD4+ cells using lentiviral vectors with regulatory sequences from the CD4 gene. Blood. 101:3416-3423.

30. Porritt, H.E., Gordon, K., and Petrie, H.T. 2003. Kinetics of steady-state differentiation and mapping of intrathymic-signaling environments by stem cell transplantation in nonirradiated mice. J. Exp. Med. 198:957-962.

31. Otsu, M., et al. 2002. Reconstitution of lymphoid development and function in ZAP-70-deficient mice following gene transfer into bone marrow cells. Blood. 100:1248-1256.

32. Bellier, B., Thomas-Vaslin, V., Saron, M.F., and Klatzmann, D. 2003. Turning immunological memory into amnesia by depletion of dividing $\mathrm{T}$ cells. Proc. Natl. Acad. Sci. U. S. A. 100:15017-15022.

33. Puisieux, I., et al. 1996. Restriction of the T-cell repertoire in tumor-infiltrating lymphocytes from nine patients with renal-cell carcinoma. Relevance of the CDR3 length analysis for the identification of in situ clonal T-cell expansions. Int. J. Cancer. 66:201-208.

34. Pannetier, C., Even, J., and Kourilsky, P. 1995. T-cell repertoire diversity and clonal expansions in normal and clinical samples. Immunol. Today. 16:176-181.

35. Williams, B.L., et al. 1998. Genetic evidence for differential coupling of Syk family kinases to the T-cell receptor: reconstitution studies in a ZAP-70-deficient Jurkat T-cell line. Mol. Cell. Biol. 18:1388-1399.

36. Unutmaz, D., KewalRamani, V.N., Marmon, S., and Littman, D.R. 1999. Cytokine signals are sufficient for HIV-1 infection of resting human T lymphocytes. J. Exp. Med. 189:1735-1746.

37. Hirschhorn, R. 2003. In vivo reversion to normal of inherited mutations in humans. J. Med. Genet. 40:721-728.

38. Lo, M., et al. 1999. Restoration of lymphoid populations in a murine model of X-linked severe combined immunodeficiency by a gene-therapy approach. Blood. 94:3027-3036.

39. Otsu, M., et al. 2000. Lymphoid development and function in X-linked severe combined immunodeficiency mice after stem cell gene therapy. Mol. Ther. 1:145-153.

40. Steinberg, M., et al. 2000. Retrovirus-mediated transduction of primary ZAP-70-deficient human $\mathrm{T}$ cells results in the selective growth advantage of gene-corrected cells: implications for gene therapy. Gene Ther. 7:1392-1400.

41. Yee, J.K., et al. 1994. A general method for the generation of high-titer, pantropic retroviral vectors: highly efficient infection of primary hepatocytes. Proc. Natl. Acad. Sci. U. S. A. 91:9564-9568.

42. Dardalhon, V., et al. 2001. IL-7 differentially regulates cell cycle progression and HIV-1-based vector infection in neonatal and adult CD4+ T cells. Proc. Natl. Acad. Sci. U. S. A. 98:9277-9282.

43. Pannetier, C., et al. 1993. The sizes of the CDR3 hypervariable regions of the murine $\mathrm{T}$-cell receptor beta chains vary as a function of the recombined germ-line segments. Proc. Natl. Acad. Sci. U. S. A. 90:4319-4323.

44. Cochet, M., et al. 1992. Molecular detection and in vivo analysis of the specific $\mathrm{T}$ cell response to a protein antigen. Eur. J. Immunol. 22:2639-2647.

45. Arden, B., Clark, S.P., Kabelitz, D., and Mak, T.W. 1995. Human T-cell receptor variable gene segment families. Immunogenetics. 42:455-500. 